\title{
Focusing on maturity levels for the effective management of end users involved in software project teams
}

\author{
A Leonard* \\ Department of Informatics, University of Pretoria \\ Pretoria 0002, Republic of South Africa \\ aleonard@hakuna.up.ac.za
}

Received October 2003

\begin{abstract}
This paper introduces a model of three maturity levels, which helps managers to evaluate end user involvement during systems development. This model is based on research done in terms of how relationships between IT departments and end users are constructed. The value of the three levels lies therein that it gives the IT department the advantage of understanding and supporting the end user in a more effective way during systems development. The model also enhances more efficient interaction between all participants of a project team. Furthermore, it gives management on the IT and business side the ability to establish training needs for both the end users and IT professionals of software project teams. End users on the other hand, who are aware of their level of maturity, could play a more efficient role during the systems development process.
\end{abstract}

*To whom all correspondence should be addressed.

\section{Introduction}

Much has been said in the literature regarding the importance of improving the performance of software development people as well as the quality of service and support they give to end users. Models have been developed to indicate that focus should be placed on the processes, technology and people in order to achieve better performance. Furthermore, it has also been pointed out that the people-focus is the component that gets by far the least attention. Most of these models show a typical starting level or point of maturity, in terms of how the processes and technology are managed and applied during systems development. They also indicate how organizations can climb the ladder to the last or highest level of maturity (Humphrey, 1990; Currtis, Hefley \& Miller,. 1995). In general, these maturity levels are based on the sophistication of the management approaches applied to manage the software process and the technology that supports it. As such, the People Capability Maturity Model (P-CMM) as described by Curtis et al. (1995) focuses on the kind of attention team-members should get from management during the systems development process. As such this model emphasizes the importance of training, compensation, skills, and motivation of the 'workforce'. 'The People Capability Maturity Model (P-CMM) focuses on continuously developing the human assets of a software or information systems organization.' (Curtis et al., 1995). In recent research done by Fok et al. (2001), they use the term TQM maturity to refer to the degree of TQM implementation in an organization. In their research they argue that the maturity of TQM can be measured by examining three dimensions: the perceived use of TQM programs; employees' perceived influence on quality issues and employees' understanding of specific TQM tools/techniques.

It is quite clear from all this research that the term maturity can be seen as some sort of indication of how well employees or organizations 'cope' with a given process. Stated differently, all these research studies have the viewpoint that a maturity level is actually established in terms of how well employees/management understand and apply the technology and the appropriate processes.

In this paper the viewpoint is held that the level of maturity is something which is determined by the given situation and not something end users or managers can decide about or emerge to. In other words, the nature and complexity of any software project at any given point in time demand certain skills and knowledge of all the participants of the project team determining the expected maturity of all participants. $\mathrm{Lu}$ (1995) states that '...information systems grow over stages because of goal displacement, organizational learning, task demands variety, personal needs, and environmental forces.'

Furthermore, it is argued that maturity is directly linked to the nature or type of relationship that exists between an IT professional and an end user (called IT-end user relationship in this paper) and as such the maturity level of an end user is determined by the degree of dependency of that end user on service and support from IT professionals. In terms of a theory of IT-end user relationships it is argued in this paper that the maturity level of an end user would indicate the end user's skills, responsibility, training needs, and degree of independence on IT professionals during system development. Such maturity levels are valuable in terms of 
determining who should form part of a project team, the nature of support end users need as well as setting up IT budgets. Furthermore, an end user who knows his/her abilities in terms of his/her maturity level and who gets support from management would be more valuable for a project team.

\section{Research approach}

An interpretive research approach was followed, taking into consideration the important principles for interpretive research as stated by Klein and Myers (1995) and Sahay, Palit and Robey (1994). Apart from doing a theoretical study of the field, the research has been complemented by the thought experiment approach in order to legitimize theories on the basis of plausibility. The motivation for making use of thought experiments is that it is actually impossible or impractical to try and conduct a 'normal' case study for the purpose of this kind of research study in the form of real experiments. A real experiment would require participants in the case study to take part in a role play over a period of at least some months or even years to experience the managerial value of the proposed theoretical model.

\begin{abstract}
'Thought experiments are not done mentally purely for the sake of convenience. They are thought experiments precisely because they cannot be performed, in principle or in practice, in the empirical world' (Introna \& Whitley, 1997).
\end{abstract}

In this research study thought experiments were used to get feedback from IT professionals and end users with regard to the working relationship between business people (non-IT workers) and IT professionals. The feedback that was obtained in this way was used to inductively refine the research results (theoretical models).

\section{Brief background on thought experiments}

A thought experiment can be defined as follows:

\begin{abstract}
'It is a coherent narrative of an unrealizable experimental situation, commensurate with the current paradigm, that is explicitly constructed in order to destroy the current paradigmatic position or to support an emerging paradigmatic position' (Introna \& Whitley, 1997).
\end{abstract}

According to Introna and Whitley (1997) the convincing power of a well-designed thought experiment cannot be disputed: 'Its ability to persuade seems to be in the use of common everyday terms and concepts. As a result, thought experiments tend to have an appeal way beyond the limitations of disciplinary boundaries.' (op. cit.). They furthermore state that although thought experiments are often seen as nice 'stories' to entertain students or add some spice to an otherwise dull lecture and that there are many reasons to believe that thought experiments do not belong in 'serious' science, there seems to be equally sufficient reason to believe that they not only belong, but could play a decisive role in advancing understanding in situations where contradiction and confusion exist.

Introna and Whitley (1997) argue that thought experiments can play a significant role in information systems research. They state that these types of experiments have the potential to bring clarity to a muddled discourse in a way that empirical research often cannot.

Introna and Whitley (1997) give important information or guidelines regarding the construction of thought experiments. They state for example that a thought experiment is explicitly constructed, within the confines of the existing paradigm, in order to destroy the existing paradigmatic position, or in order to construct an argument for a new position.

Introna and Whitley (1997) furthermore argue that thought experiments could be either destructive or constructive. Destructive thought experiments can be considered as directed against an existing theory and are designed to undermine the theory by demonstrating either an inconsistency internal to the theory or its incompatibility with other background beliefs. In contrast, constructive thought experiments are directed at providing support for a contested existing theory or argument, directed at clarifying a known and emerging argument, or can be an occasion for speculation to open up the debate concerning an emerging theory or argument.

\section{Defining and IT-end user relationship}

According to Leonard (2002) an IT-end user relationship consists of two dimensions, namely a physical dimension and an abstract dimension. The physical dimension describes those elements that are necessary in order to enable contact between IT and its end users, whereas the abstract dimension describes the soft issues (such as trust, commitment) of a relationship. These two dimensions enable one to fully describe the holistic nature of such a relationship and encapsulate the important elements of a support-oriented organization, namely mutuality, belonging, and connection, as mentioned by Pheysey (1993) in her book Organizational Cultures. The basic components of such a relationship are illustrated in Figure 1.

Without going into all the detail of the different elements of the physical and abstract dimensions, the paper focuses on the end user as a role player during systems development and the different types of relationships end users could be engaged in during service and support activities provided by IT professionals. Based on this discussion IT-end user relationship types are defined which could be linked directly to maturity levels of end users. 
R-E-L-A-T-I-O-N-S-H-I-P

IT Department

End User

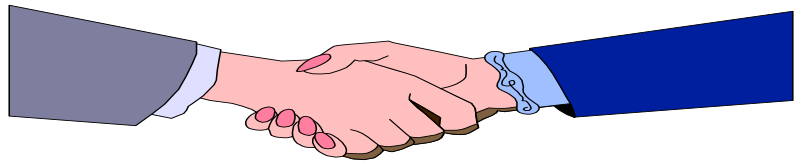

\begin{tabular}{|c|c|c|}
\hline Physical Dimension & Abstract Dimen & \\
\hline Procedures & Dynamic & Sensitive to Change \\
\hline People & Knowledge Base & Supportive Culture \\
\hline Structures & Holistic nature & \\
\hline Technology & Commitment & $\begin{array}{l}\text { Co-operation } \\
\text { Sustainability }\end{array}$ \\
\hline
\end{tabular}

Figure 1: The basic components of an IT-end user relationship (Leonard, 2002)

\section{End user characteristics}

Leonard (1998) state that end users are normally regarded by IT professionals as the inferior party and as such they do not give end users the opportunity to become 'fully' involved in system development project activities, apart from allowing them to give guidance in terms of their basic needs.

Bannon (1991) argues that viewing computer users as naive is bad enough, viewing them as idiots is even worse. Apart from the values question that arises, there are clear design implications if this faulty view of users is implicitly adopted. Just because users do not understand how the machine works or have difficulty with the system designer's terminology, does not imply that they are stupid, as some developers apparently conclude, if we are to judge from the systems that are at times designed. According to Bannon (1991), the system design team should start out with the 'understanding that workers/users are competent practitioners, people with work tasks and relationships which need to be taken into account in the design of systems and with whom they must collaborate in order to develop an appropriate computer system.' (op. cit.).

The idea that we must design systems so that 'any idiot can use them' bears close scrutiny. Taking this as a serious design goal can often result in systems that necessarily produce such stupid behaviour by end users. A typical consequence is that an incredible amount of 'intelligence' must go into its initial design and maintenance. Taken to the extreme, we have the prospect of artificially intelligent systems operated by morons - an absurd scenario.

'Man is one of the best general-purpose computers available and if one designs for man as a moron, one ends up with a system that requires a genius to maintain it. Thus we are not suggesting that we take man out of the system, but we are suggesting that he be properly employed in terms of both his abilities and limitations' Bannon (1991).

Fortunately, in recent years this particular problem seems to have diminished, probably as a result of designers and others finally developing a better understanding of the end user's perspective. Certainly, there are some applications where a minimal interface that is easy to learn is desirable, but this is not usually the case when dealing with systems that will be used by people in their everyday long-term work. In conclusion, Bannon (1991) states that we need to pay attention to the capabilities of the system and allow users greater flexibility and expressiveness in their use of it.

In terms of a theory of IT end user relationships it is argued that in order to ensure the establishment of sound IT-end user relationships, information systems development should move towards approaches concerned with sense-making and argumentation rather than control, and end users can no longer be 'passive' role players during systems development. Furthermore, the term 'end user' could be in dispute in terms of IT-end user relationships where the end user becomes an integral part of the team (Leonard, 1998).

\section{Research results obtained from thought experiments}

In this section the author analyse the feedback on questions posed to practitioners from both the public and private sectors. The purpose of the questions was multi fold. First of all the author wanted to get clarity on the approaches followed in establishing and maintaining sound relationships between IT professionals and end users, whilst the second question was focused on the factors affecting the performance of project teams. Another important aim was to get feedback in terms of proposals on how to understand and support the end user in a more effective way during systems development as well how to enhance more efficient interaction between all participants of a project team. 
The participants who took part in this case study are people who act either as IT professionals in their fields or as end users.

The responses received from the participants are given verbatim. In other words, no changes have been made to the grammar of the feedback. Only spelling mistakes were corrected. Furthermore, only those parts of groups' responses that could be regarded as of interest to the phenomenon under investigation, are cited.

\section{Feedback from group 1}

- $\quad$ 'To have a relationship, communication is of utmost importance...Man being an entity searching for maximum output from minimum input, wants communication to be as easy as possible. We can all do with less frustration, stress and effort.'

\section{Conclusion(s):}

These remarks clearly indicate the frustration of end users where communication facilities and/or structures are not functioning effectively. Furthermore, it emphasizes the importance of proper communication procedures and facilities for the establishment and maintenance of sound relationships.

- 'Whatever the relationship between the end user and IT, information technology has become a tool without which one can no longer exist in this competitive world. The balance between the frustration of this non living, living thing and the results obtained is definitely in favour of IT.'

\section{Conclusion(s):}

This citation not only indicates the importance of 'working relationships' between IT professionals and its end users, but also regards information technology as an indispensable piece of 'equipment' or 'tool' which is necessary in order to support all participants in addressing the needs of end users or business units. The last part of the citation clearly relates to end users' negative perception of the culture that exist in many IT departments, namely that IT professionals play the dominant role.

- 'The intelligence of the people should never be underestimated. Man has the ability to manage any obstacle if he knows what the objective is. Through innovative thoughts every person can mould a system to suit his needs. This freedom within certain limits should therefore be given to the end user.'

Conclusion(s):

This citation clearly emphasizes the importance of regarding the end user as a intellectual and knowledgeable participant in any relationship and as such IT-end user relationship environments should 'allow for active end users').

\section{Feedback from group 2}

- 'People do not take time to communicate some of the reasons might be that they are too busy due to work overload, or they might want to keep information to themselves. Not to communicate means that experience and assistance are not shared and work duplication is caused.'

Conclusion(s):

This citation again emphasizes the importance of proper communication procedures and facilities between IT and its end users. It furthermore emphasizes the importance of a supportive culture between IT professionals and its end users.

\section{- $\quad$ Possible Actions}

1. Introduce informal 'Information Sharing' sessions during office hours.

2. Get to know your colleague. If you know a person well it will be easier to communicate with them.

3. Train the user to help themselves where there are not really problems but situations,

4. Allow users to work and rotate in IT Department. Have special training schedules distributed within the organization to invite users to learn IT related skills.

5. One 'Marketing' Person must be appointed to have regular standing meetings, over and above departmental meetings, with key people in the user departments'.

\section{Conclusion(s):}

This citation forms part of the group's recommendations of what should be done in order to improve the unsatisfactory situation in terms of poor relations between IT and its end users. Apart from the fact that end users have to be trained, it recommends two important aspects, namely the sharing of information about what IT is doing for the business and secondly, the marketing of what and how IT can support the business. In other words, participants of any given IT-end user relationship should share their progress and what they are doing with the rest of the organization and should also take responsibility in the marketing of their service and support. This will ensure that everyone that might be interested in what the participants of an IT-end user relationship is doing, can get involved if necessary. This will especially be of relevance in large organizations and will surely contribute in narrowing the 'culture gap'. The sharing of information amongst other employees regarding what is going on in the organizational environment is a very relevant and important issue. Kober and Knowles (1996) state that the nature and role of information must change from being restricted and used for power to being openly shared, in abundance, with everyone in the system. According to Kober and Knowles (1996) 'it needs to be available to all of us like the air we breathe.' (op. cit.). 


\section{Feedback from group 3}

- 'Management must promote a culture where people feel free to contribute their ideas, where involvement in problem solving and decision making is the norm.'

\section{Conclusion(s):}

It is clear that the need for the establishment of a culture where people can 'freely' contribute (getting involved) is seen as an important element in the creation of a supportive culture and in the establishment of a self-organizing environment. This issue is also addressed in the previous group's last citation (cf above).

- '...The end-user must feel comfortable with the equipment/tools at his disposal. He must know where to find help when he has a problem. He must be well prepared and timeously trained for the introduction of new software. There must be end-user liaison between the technical interfaces and the various service providers.'

\section{Conclusion(s):}

The citation strongly debates in favour of certain important elements which could ensure the acceptance of software products developed by the IT department for the business. Apart from emphasizing proper training and support for end users regarding the use of any proposed new system, the citation strongly argues for co-operation, involvement and a supportive culture between IT and its end users.

- '... the end-user must be receptive to change. He must be able to provide and actively participate in training. He must be capable of submitting systems service requests. The end-user must also be pro-active.'

\section{Conclusion(s):}

The citation not only argues for end users to be active participants, it also emphasizes the fact that this kind of behaviour can only be expected from end users if the necessary environment (culture) is created. Furthermore, although it states in general that end users should be receptive to change, it could be argued that in terms of the previous citations and within an ITend user relationship environment it is also important to support participants in this regard.

\section{Feedback from group 4}

- 'Something that is definitely damaging relationships between IT and the end users, is when IT starts developing a system without proper consultation and when the product is finished, it is not what the end user wants...The IT personnel feel that the training of the end users is not up to standard and that this is to the disadvantage of communications between the two groups (IT professionals and end users)...In order to establish sound relationships, IT must train its end users properly before they install new software (and not a year after installation). End users should also be kept up to date regarding any changes that are made to the system.'

\section{Conclusion(s):}

First of all this group regards the lack of end user involvement during the systems development life cycle as one of the major contributors to the damaging of ITend user relationships. The proper training of end users is regarded as an important element in the establishment of sound relationships. In terms of the IT-end user relationships, it emphasizes the importance of a proper knowledge base and as such a supportive culture.

\section{Summary of conclusions}

From the abovementioned feedback the following aspects are relevant and of importance in terms of how relationships between IT professionals and end users can be improved and a clear indicator that the 'maturity' of end users should be taken into consideration when teams are managed.

- All of the groups emphasized the importance of end user training and the creation of a proper knowledge base in order to ensure sound communications.

- Group 1, group 2 and group 4 emphasized the importance of informing the end user about the progressing of information systems projects. Group 1 pointed out that in order to ensure that IT-end user relationships are on a sound basis in general, IT should also communicate project progress to those users 'outside' a specific relationship. Group 4 called it 'information sharing'. Group 4 also mentioned the importance of this, but suggested that an IT 'marketing person' should be responsible for this task. This also link directly to the notion of maturity levels - the higher the level of maturity the easier the communication and 'understanding' of the end user will be regarding 'how are things progressing?'.

- The different groups mentioned many of the important elements of the physical and abstract dimensions as indispensable for the establishment and maintenance of sound relationships between IT and its end users. Group 1, for example, discussed the important role that commitment plays and especially pointed out that the commitment of all parties in a development team is necessary for achieving success.

- Group 3 pointed out that the IT professionals should do a follow-up on the service and/or support they gave the end user. This they regard as necessary, because often the end user 'thinks' he/she now understands how to do or perform a certain task or how to cope with a given situation, just to find out (after the IT professional has left) that he/she still needs support. Their standpoint emphasizes the importance of the 'follow-up' ( $c f$ group 3 ) which is again narrow related to the level of maturity.

In the next section theories of maturity levels are briefly described where-after a theoretical framework for end user maturity levels in an IT-end user relationship environment is described and illustrated. 


\section{Relationship types and maturity levels of end user involvement}

From the theories of Ciborra (1993), Klepper (1995) and Kumar and Van Dissel (1996) it follows that there could be a large variety of arrangements that could be used to define how people can work together under specific circumstances. It is argued that the kind of contractual arrangement (to use the words of Ciborra (1993) needed to define the different types of IT-end user relationships should be based on three broad types, namely those which link the two parties very closely, those that maintain a distance, and those that fall in between these two situations. These three types of IT-end user relationships will be used to formulate the theory of maturity levels of end users, as discussed in the rest of the paper.

On the basis of the abovementioned, and in terms of the first two broad types of relationships ( $c f$ above), namely those which link the two parties very closely and those that maintain a distance between two parties, IT-end user relationship types of this kind should be defined as:

- $\quad$ relationships where the end user is highly dependent on IT for support. They are referred to as hard relationships, where hard indicates a high frequency of communication (contact) between the parties because of the end user's inability (lack of knowledge) to help himself. This relationship therefore defines a maturity level of end user involvement which is referred to as a low maturity level. This implies that the given project or service IT has to provide does not expect end users to have any specific technical knowledge or skills;

- $\quad$ relationships where the end user is highly independent on IT for support. In this research study they are referred to as soft relationships, where soft indicates a low frequency of communication (contact) between the parties because of the end user's ability (knowledge) to help himself. This relationship therefore defines a maturity level of end user involvement, which is referred to as a high maturity level. This implies that the given project or service expects a high level of technical knowledge or skills from the end user(s).

These two types of relationships help us to address those environments where end users are on the one hand (totally) dependent upon the IT department's service and support in order to develop and/or maintain a certain software product, whereas on the other hand they are (mostly) independent of service and support from the IT department for the development and maintenance of a specific product. Although the former (hard relationships) are aimed at situations where the kind of technical expertise is in the hands of the IT department, for example in the case of a mainframe environment, the latter is aimed at situations where the end user has enough knowledge to be able to cope with a situation without much support from the IT department. In such a case one can think, for example, of end users using a software package to build their own end user system for their office environment needing only the IT department's support for a short period of time to interface with other systems (cf above). In other words, relationship types are primarily based on the degree of independence with which end users can make use of the 'essential technologies' available to them to develop or maintain software products for the organization. Therefore, it is possible that an end user may want to develop an end user system for an office environment making use of normal end user computing tools, but, because of a lack of knowledge, the end user asks the IT department to undertake the development and maintenance responsibility. Such an end user will be involved in a hard relationship ('close relationship', cf Klepper's theory) with the IT department. Another example of a soft relationship in a mainframe environment is when an end user builds an information retrieval system, making use of a software productivity tool, like Easytrieve, which the end user knows well. Such an end user needs little service and support (maybe permission to access certain files) from the IT department in order to create such a system.

It could furthermore be argued that it is not true that if an end user is not 'totally' dependent on the IT department for a certain service, then the end user is 'totally' independent of the IT department, and can therefore use the 'essential technologies' without support. Many end users, especially those who keep themselves busy with end user computing activities, are still in a process of becoming literate and familiar with all the tools and therefore are neither in a 'soft' nor a 'hard' relationship environment (q.v. Richard Nolan's assimilation theory of technology in organizations). For this reason it would be reasonable to introduce a third relationship type in order to accommodate those end users who fall between these two extremes and this is called a neutral relationship type, which defines a neutral maturity, level of end user involvement.

Based on this research study, Figure 2 illustrates the relationship between relationship type and maturity level.

It is important to note that end users who are involved in the soft relationship environment are normally to a large extent dependent on the IT department as far as the setting of the necessary infrastructure (the buying of software packages, the implementation of network facilities, training, help desk facilities, etc.) is concerned. In some cases the responsibility for the acquisition of hardware and software for computing in a specific end user systems environment may be delegated fully or partially to those end users, but the end user still needs to obey the standards set by the IT department (Schultheis \& Sumner, 1995). Therefore, the term 'totally independent' should be seen in relative terms. 


\begin{tabular}{|c|c|c|c|}
\hline $\begin{array}{c}\text { Maturity } \\
\text { level } \\
\text { Relationship } \\
\text { type }\end{array}$ & Low & Neutral & High \\
\hline $\begin{array}{c}\text { Hard } \\
\text { (high frequency) }\end{array}$ & $\begin{array}{c}\text { 'No' Technical } \\
\text { Skills/Knowledge }\end{array}$ & \\
\hline Neutral & & $\begin{array}{c}\text { Some Technical } \\
\text { Skills/Knowledge }\end{array}$ & \\
\hline $\begin{array}{c}\text { Soft } \\
\text { (low frequency) }\end{array}$ & & & $\begin{array}{c}\text { Advanced Technical } \\
\text { Skills/Knowledge }\end{array}$ \\
\hline
\end{tabular}

Figure 2: Maturity matrix for end user involvement

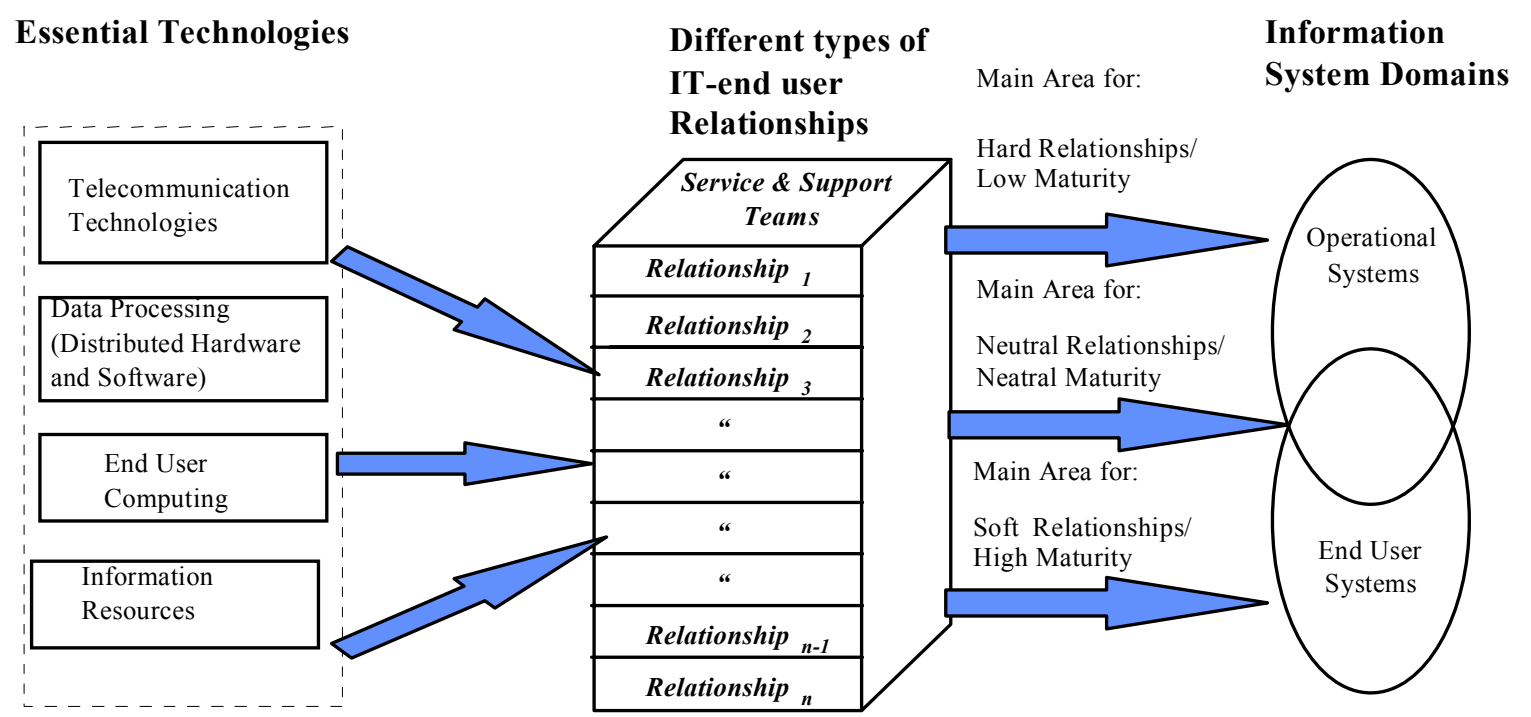

Figure 3: Relationship types and end user maturity levels in an IT-end user relationship environment (based on the work of Sprague \& McNurlin (1993), Gunton (1990) and Di Carlo (1989))

The abovementioned IT-end user relationship types may be present in any of the information system domains ( $c f$ Figure 3) which implies that maturity levels expected of end users depend on the nature and complexity of the given systems development situation. However, the main area in figure 3 indicates the dominant relationship type.

\section{Practical implications of this research}

The implications of this research for organizations are three fold. Firstly, IT managers should be aware of the importance of having the right people (end users and IT professionals) on software project teams. In terms of an ITend user relationship, managers must ensure that the 'right' end users take part in the relationship. This is sanctioned by Woodcock and Dave (1989):

'Successful managers understand the vital importance of getting the best possible candidates into ... jobs.'
Having the 'right end user' involved in an IT-end user relationship ${ }^{1}$ cannot be over emphasized, because if end users are not aware of the damage and problems that the 'wrong' delegate may cause in a relationship, not only can the soundness of the relationship as such be in danger, but proper service and support are at stake. The reason for this is actually quite clear: one of the important components of a relationship, namely effective communication will not be possible.

This will mean (in the normal sense of the word) that the user will have the necessary financial and other powers from the side of the specific end user systems environment to act on behalf of the specific business environment. In this regard Orlikowski (1992) remarks:

\footnotetext{
${ }^{1}$ The delegation trap and right end user are related issues and the importance thereof have been explained by Gunton (1988) and Roode and Smith (1989) respectively.
} 
'...power enters into human interaction through providing organizational capabilities for humans to accomplish outcomes'

In general one could argue that to have the right people on a team can only enhance sound relationships and as such sound interaction between participants of a team. This is sactioned by Jiang, Motwani and Margulis (1997) on how groups influence their members) who emphasizes that team effectiveness is strongly affected by members' interactions. Secondly, it emphasizes the importance of having the organization and the IT department properly aligned. The elements of both the physical and abstract dimensions play a critical role during the aligning process between IT department and the organization (business). 'Each of these elements plays a specific social role in an IT-end user relationship environment, which impacts on the soundness of such a relationship as well as the success of alignment between IT and the business' (Leonard 2001). As such proper alignment between IT and the business supports readiness for any level of maturity, which means an organization has the manpower with the necessary skills and abilities to apply the technology to achieve its goals. If this is not the case, organizations could often find themselves in the uncomfortable situation of not being able to reap competitive advantage from information technology.

Thirdly, end user maturity levels, as introduced in this paper, differ from current thinking in the sense that the level of maturity of an end user is based on what the given project or service/support situation expects from end users in terms of skills and knowledge. In other words, how efficient will the end user be and how well can the end user cope with the given situation and to what extent will he/she be dependent on support from IT professionals? It is argued that an answer to this question lies in the identification of the maturity level of the end user.

Although mainframe projects would normally place end users in a very dependent position, and as such on a low level of maturity, it is clear that with the development of productivity tools, especially on the terrain of web-based application development, this situation can change drastically in the future. These levels of end user maturity can therefore serve as an important 'measure' or indication of training needs, software that is needed, the amount of support end users in the organization need and as such, how IT development will impact on the organizational budget. It could even be seen as a measurement of productivity for business units, which is a field for further research.

\section{Concluding summary}

In this paper IT-end user relationships are defined and three types of these relationships are introduced, which accommodate end users according to the degree of their independence of the IT department in getting service and support for applying the 'essential technologies'. Furthermore, the paper introduces the concept of maturity levels for end users. The paper argues that the expected maturity for participants of any service or support activity offered by the IT department is determined by the given situation. As such, all participants of a project team need to have certain skills and knowledge to accomplish a given task as a team. In this paper it is argued that the maturity levels of end users are directly linked to the type of relationship existing between an IT professional and an end user and as such it is determined by the degree of dependency of an end user on service and support from IT professionals. In this regard three levels of maturity for end users is introduced, namely a high level maturity which is linked to a relationship of a soft type, a low level maturity which is linked to a relationship of a hard type and a neutral level maturity which is linked to a relationship of a neutral type. This approach towards maturity levels differs largely from existing thinking. Research studies of existing models held the viewpoint that a maturity level is actually established in terms of how well employees/management understand and apply the technology. In this paper an opposite viewpoint is held, namely that a maturity level is a requirement which is determined by the nature of the service and support. This way of thinking therefore implies that project managers can determine the maturity levels of all their IT projects beforehand and as such do better planning in terms of what knowledge and skills are to successfully accomplish a given project. Furthermore, project managers will be in a position to ensure that end users get involved more effectively.

\section{References}

Anderson, E. \& Weitz, B. 1989. 'Determinants of continuity in conventional industrial channel Dyads', Marketing Science 8(4):310-323.

Bannon, L.J. 1991. 'From human factors to human actors.' In Greenbaum, J. \& Kyng, L. The role of psychology and human-computer interaction studies in system design. UK: Addison-Wesley.

Ciborra, C.U. 1993. Teams, markets and systems: Business innovation and information technology. Cambridge, UK: Cambridge University Press.

Curtis, B., Hefley, W.E. \& Miller, S.A. 1995. People capability maturity model (P-CMM). UK: Addison-Wesley.

Desanctis, G. \& Poole, M.S. 1994. 'Capturing the complexity in advanced technology use: Adaptive Structuration Theory', Organization Science 5:121-147.

Di Carlo, P. 1989. 'Information systems management', Telecommunications, October:49-58.

Fok, L.Y., Fok, W.M. \& Hartman, S.J. 2001. 'Exploring the relationship between total quality management and information systems development', Information \& Management, 38:355-371.

Giddens, A. 1984. The constitution of society. Berkeley: University of California Press.

Gunton, T. 1990. Business information technology: Inside information technology: A practical guide to management issues. London: Prentice Hall. 
Humphrey, W.S. 1990. Managing the software process. Reading, Massachusettes: Addison-Wesley Publishing.

Introna, L.D. \& Whitley, E.A. 1997. 'Imagine: Thought experiments in information systems research.' In Proceedings of the IFIP TC8 WG 8.2 International Conference on Information Systems and Qualitative Research, Philadelphia, Pennsylvania, USA, pp. 481-496.

Jiang, J.J., Motwani, J. \& Margulis, S.T. 1997. 'IS team projects: IS professionals rate six criteria for assessing effectiveness', Team Performance Management, 3(4):236243.

Klein, H. \& Myers, M.D. 1995. 'The quality of interpretive research in information systems'. Unpublished document.

Klepper, R. 1995. 'Outsourcing relationships'. In Khosrowpour, M. (Ed.). Managing information technology investments with outsourcing. Harrisburg, USA: Idea Group Publishing.

Kober, K. \& Knowles, R. 1996. 'Measurement in selforganizing systems', Journal for Quality and Participation, January/February: 38-41.

Kumar, K. \& Van Dissel, H.G. 1996. 'Sustainable collaboration: Managing conflict and cooperation in interorganizational systems', MIS Quarterly, September:279-300.

Leonard, A.C. 1998. 'Information technology-end user relationship in a changing environment'. Unpublished D.Com.(Informatics) thesis, University of Pretoria.

Leonard, A.C. 2001. 'The importance of the IT-end user relationship paradigm in obtaining alignment between IT and the business'. In Papp, R. (Ed.). Strategic information technology: Opportunities for competitive advantage. London: Idea Group Publishing, pp.218-236.

Leonard, A.C. 2002. 'A conceptual framework for managing relationships between all participants during IT service and support activities', South African Journal of Industrial Engineering, 13 (2):81-96.

Lu, H. 1995. 'Managerial behaviours over MIS growth stages', Management Decision MCB, 0025-1747, 33(7):4046.

Orlikowski, W.J. 1992. 'The duality of technology: Rethinking the concept of technology in organizations', Organizational Science: 399-427.

Pheysey, D.C. 1993. Organizational cultures. New York: Routledge.

Roode, J.D. \& Smith, A.J. 1989. 'User involvement in systems development', South African Journal of Economic and Management Sciences 2:7-20

Sahay, S., Palit, M. \& Robey, D. 1994. 'A relativist approach to studying the social construction of information technology', European Journal of Information Systems 3(4):248-258.

Schultheis, R. \& Sumner, M. 1995. Management information systems: The manager's view. USA: Irwin Inc.

Sprague, R.H. \& McNurlin, B.C. 1993. Information systems management in practice. (3rd Edition), Englewood Cliffs: Prentice Hall.

Woodcock, M. \& Dave, F. 1989. Clarifying organizational values. Englewood Cliffs: Prentice Hall. 
